\title{
MODELING PULL-OUT TEST OF DENTAL IMPLANTS
}

\author{
Anna Dollar', Kevin P. Meade ${ }^{2}$ \\ ${ }^{1}$ Manufacturing and Mechanical Engineering Department, \\ Miami University, Oxford, $\mathrm{OH}$, USA \\ ${ }^{2}$ Mechanical, Materials and Aerospace Engineering Department, \\ Illinois Institute of Technology. Chicago, IL, USA
}

\begin{abstract}
The objective of this paper is to investigate bone-implant interface failure using analytical techniques of fracture mechanics. The implant usually is anchored to the surrounding bone by growth of bony tissue into the surface of the implant. A mechanical interlock is formed between the implant and the bone. Plane strain conditions are imposed. By using a continuous distribution of edge dislocations to represent interfacial debonding, the problem reduced to a system of singular integral equations that was solved numerically using standard collocation techniques. Quantities of interest are the extent of the debonded zone, the relative displacement between the implant and the bone and the stresses at the bone-implant interfaces, all of which depend on the load in a nonlinear fashion.
\end{abstract}

Biomed Eng Appl Basis Comm, 2003 (August); 15: 133-142.

\section{INTRODUCTION}

Dental implants are becoming an increasingly popular alternative treatment for replacing missing teeth. Instead of using a bridge that spans between tceth, a permanent replacement tooth is attached to an implant in the mandible or maxilla.

In this study, dental implants of the ingrowth type are considered. These implants rely on bony ingrowth for fixation. Typically they are coated with hydroxyapatite that helps the formation of interfacial bone adjacent to the implant [1]. To install the implant, a hole slightly smaller than the outside diameter of the implant is drilled in the mandible or maxilla. The implant is then press-fit into place and covered with soff tissue until healing is completed. This creates a

Received: May 28, 2003; Accepled: June 14, 2003 Correspondence: Anna Dollar, Professor Manufacturing and Mecharical Engineering Department, Miami University, Oxford. OH, USA. E-mail: dollara@muohio.edu state of stress resembling remote compression. At the end of the healing period the implant is uncovered and inspected. If the implant interface has healed properly, the bond between the bone and the implant is capable of supporting load levels typically seen during mastication. The replacement tooth is then fastened to the implant with a small screw.

Over time, the gumline will recede somewhat, exposing some of the implant. This resorption is apparently due to a stress shiclding effect caused by the implant. However, this does not constitute a failure of the implant. The implant is considered to have failed if the initial bond did not form properly and a prosthetic tooth could not be installed, or if it becomes loose after the installation of the tooth. In both cases the implant would have to be rcvised, causing the patient to undergo the entise procedure again.

A significant problem with implants is that they can loosen and cause severe pain, necessitating surgical revision [2-3]. Although the exact mechanism of loosening is unknown, the debonding (fracture) of the bone-implant interface may be the controlling factor in this process. The strength of this interface depends on factors such as cancellous bone structure 
and surface preparation, press-fit used and geometry of the implant. However, a quantitative measure of the relative importance of these factors does not exist.

There is interest in understanding how and why dental implants fail, as well as in reducing the healing time. Both require ex vivo pull-out testing of recovered dental implants from controlled animal studies. For example, in [4] authors used pull-out force to assess biomechanical strength of the bond between the implant and the surrounding bone. This was used as an index of osseointegration. However, the specific details of the implant interface failure were not studied in detail.

In the mechanics of fiber reinforced composite materials, fiber push-in and pull-out tests are used for deducing the fiber-matrix interfacial strength. An analogous approach could be applied to deduce information on the bone-implant interface. In order to analyze the results of such tests a suitable mathematical model is needed. Nonlinear fracture mechanics is one logical technique to analyze this problem, but existing analytical models are inadequate to describe the nature of the process of implant loosening. An adequate modeling of failure processes is of primary importance, so that changes in bonding techniques can be evaluated on a rational basis.

The purpose of this study was to investigate the relative importance of the geometric parameters of the implant (height, diameter, etc.) and the distal surface debonding length on the gap formation along the vertical interface between the implant and the host bone. To better represent the effects of the interface, a theoretical model was used to perform a parametric study rather than employing a finite element model as in [5]. There the authors created a two-dimensional axisymmetric finite element model to study the effects of varying the implant geometry on the stress distribution along the implant interface. They noted that because of the lack of a better interface model, the conclusions from their shear stress calculations were limited.

\section{DESCRIPTION OF THE MATHEMATICAL MODEL AND PROBLEM STATEMENT}

A two-dimensional plane elasticity model is employed in a manner similar to that for studying pullout/push-out tests done in fiber reinforced composites [6]. The primary differences between the present model and the fiber reinforced composite model arise from the boundary conditions representing the implant/bone interfaces.

The bony ingrowth establishes a mechanical interlock between the two surfaces. Interface failure is expected to be primarily tensile since the interdigitation resists shear slip and relative displacements normal to the interface could occur. For minimal normal displacement there would be no relative shear displacement along the entire interface. As the interface opens to a magnitude greater than the interdigitation height, a shear-stress-free zone will form. Therefore, the conditions at the interface are no resistance to tensile stress and no relative slip until the opening at part of the interfaces becomes larger than the interdigitation height. After that, relative slip is permitted at that portion of the interface. In the present study only the first scenario is being investigated in order to assess the feasibility of the second one.

Although this research aims to better understand the debonding mechanism at the interface of two different materials, differences in the constituents' elastic moduli have been ignored. This assumption substantially simplifies the analysis. Nevertheless, it should be pointed out that the moduli of the bone and the implant are not so different, at least as compared with some composite materials, in analysis of which this simplification is often used as well. In addition, this allows us to focus on the effect of the interface properties alone. In [7] the authors report the results of a finite element analysis of a dental implant subjected to axial and lateral loading for a range of bone density distributions. They found that a sixteen fold reduction in the elastic modulus of the bone around the neck of the implant reduced the peak stress by only a factor of two. This shows that accounting for differences in the moduli of the bone and implant may not result in dramatically different results from the homogeneous model. This provides further justification for our model.

The model pull-out test is shown schematically in Figure 1. The half-plane lying in the domain $y>0$ is composed of two homogeneous isotropic elastic regions; the implant (inclusion) occupying $|\mathrm{x}|<a$ and $y<\mathrm{H}$, and the surrounding bone (matrix). It is

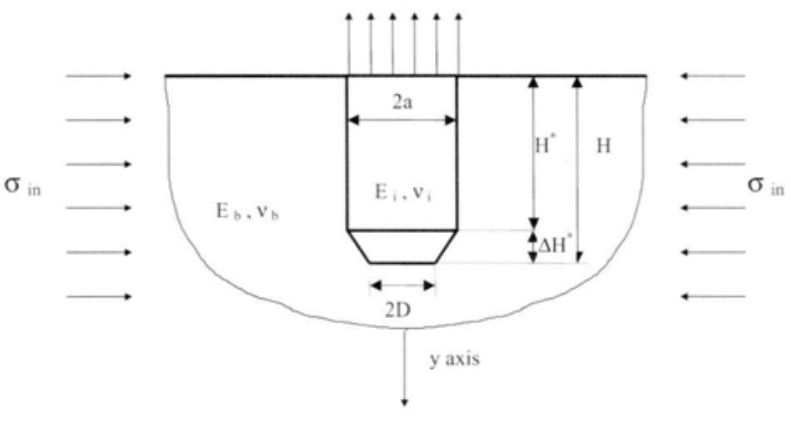

Fig. 1 Implant geometry and loading. 
assumed that both possess identical elastic constants, i.e., shear modulus $G$ and Poisson ratio $v$.

To simulate the presence of some pre-existing compression at the interface, a uniform lateral compression $\sigma_{x x}=-\sigma_{\text {in }}\left(\sigma_{\text {in }}>0\right)$ is imposed on the half-plane. Finally, the half-plane is subjected to a uniform normal traction $p$ applied along $-a<x<a$, $y=0$. This is intended to approximate the loading in a pull-out test.

Before the traction $p$ is applied, there exists a perfect bond between the inclusion and the surrounding matrix. As the traction $p$ is increased monotonically, the stress normal to the vertical interface reduces. When the normal stress reaches zero, the interface fails, i.e., opens. Bccause of the mechanical interlocking from the ingrowth of the bone, the failed interface is modeled as a no-slip interface. That is, the normal stress on the interface is zero, while no relative tangential slip of the interface surfaces is permitted. This is a similar approach to that of [8-10].

At a depth of $y=H$, a no-slip crack of half-length $D$ is introduced. This simulates a possible failure of the bond, or a lack of establishing a bond at the bottom of the inclusion. The no-slip condition is used to simulate the mechanical interlocking due to the ingrowth of the surrounding bone. In general $D$ can be equal to or smaller than a, reflecting the fact that implants are either cylindrical or have tapered ends. In the case of a tapered end there are usually holes drilled on the conical surface allowing for bone ingrowth to prevent debonding in that region. In such cases the vertical interfaces will be treated as perfectly bonded for $\mathrm{H}^{*}<\mathrm{y}<\mathrm{H}$, and the horizontal interface for $D<|\mathrm{x}|<a$. The cases of $D$ ranging from zero (no horizontal crack present) to $D=a$ will be investigated.

At any instant in the loading history, either sticking or opening occurs at a generic point along the vertical interfaces. These conditions are expressed mathematically on the vertical interface by:

stick condition: $\sigma_{\mathrm{xx}}<0, v^{*}=0, \mathrm{u}^{*}=0$

open condition: $\sigma_{x x}=0, v^{*}=0, \mathrm{u}^{*}>0$

and on the horizontal crack by:

$$
\sigma_{\mathrm{yy}}=0, v^{*}>0, \mathrm{u}^{*}=0
$$

wherc,

$$
\begin{aligned}
u^{*}= & \lim [u(a+\varepsilon, y)-u(a-\varepsilon, y)] \\
\varepsilon \rightarrow 0^{+} & \\
v^{*}= & \lim _{\varepsilon \rightarrow 0^{+}}[v(x, H+\varepsilon)-v(x, H-\varepsilon)]
\end{aligned}
$$

In these equations $\sigma_{x x}, \sigma_{y y}$ denote the Cartesian components of stress and $u$ and $v$ denote the $\mathrm{x}$ - and $\mathrm{y}$ components of displacements, respectively.

\section{METHOD OF SOLUTION}

The solution to a normally loaded, perfectly bonded half-plane is superposed with a solution due to a distribution of edge dislocations along the horizontal crack and vertical openings in an elastic half-plane.

The stress components are normalized by the applied traction $p$. The spatial variables, the opening length $L$, the crack location $H$, and the crack halflength $D$ are normalized by the implant diameter a. The dimensionless parameters of the geometry of the problem are: $h=H / a, d=D / a, l=L / a$ as shown in Figure 2.

The stresses in a perfectly bonded half-plane are:

$$
\begin{aligned}
- \text { on } x= & \pm 1 \\
& \sigma_{x \mathrm{r},}=-\sigma_{\infty}+\left[\frac{1}{2}-\frac{1}{\pi}\left(\tan ^{-1} \frac{y}{2}-\frac{2 y}{y^{2}+4}\right)\right] \\
- \text { on } y= & h \\
& \sigma_{v x p r}=\frac{1}{\pi}\left[\frac{\pi}{2}+\tan ^{-1} \frac{1-x}{h}\right. \\
& \left.-\tan ^{-1} \frac{h}{1+x}-2 h \frac{x^{2}-h^{2}-1}{\left(x^{2}+h^{2}-1\right)^{2}+4 h^{2}}\right]
\end{aligned}
$$

It is important to note that the pairs of dislocations are distributed symmetrically along the interface as well as along the horizontal crack. The interface refers to the line $x=+1, \mathrm{y}<h^{*}$; the crack refers to $|x|<d$, $y=h$.

The dislocation solution is expressed in terms complex stress potentials $\Phi$ and $\Psi$ nintroduced in [11] that are related to the Cartesian components of stress and displacements according to:

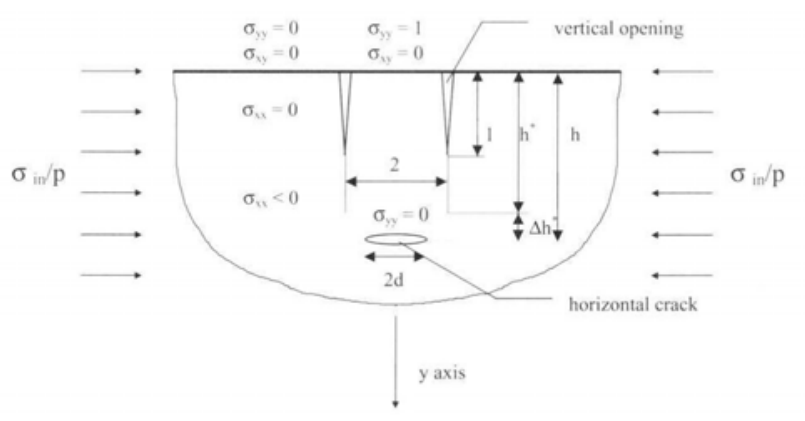

Fig. 2 Model problem. 


$$
\begin{aligned}
& \sigma_{x x}+\sigma_{y y}=2\left(\Phi^{\prime}+\overline{\Phi^{\prime}}\right) \\
& \sigma_{y y}-\sigma_{x x}+2 i \sigma_{x y}=2\left(\bar{z} \Phi^{\prime \prime}+\Psi^{\prime}\right) \\
& 2 G(u+i v)=K \Phi-z \overline{\Phi^{\prime}}-\bar{\Psi}
\end{aligned}
$$

where $\Phi$ and $\Psi$ are analytic functions of the complex variable $z=x+i y$, the prime' denotes differentiation with respect to $z$, the overbar denotes complex conjugation, and $k=3-4 v$ in plane strain.

Stress potentials for one dislocation with Burger's vector $\boldsymbol{b}=b_{x} \boldsymbol{i}+b_{y} \boldsymbol{j}$. located at a point $\mathrm{z}_{0}$ in the halfplane are given by:

$$
\begin{aligned}
\Phi^{\prime}= & \frac{\alpha}{z-z_{0}}+\frac{\bar{\alpha}\left(\bar{z}_{0}-z_{0}\right)}{\left(z-\bar{z}_{0}\right)^{2}}-\frac{\alpha}{z-\bar{z}_{0}} \\
\Psi^{\prime}= & \frac{\bar{\alpha}}{z-z_{0}}+\frac{\alpha \bar{z}_{0}}{\left(z-z_{0}\right)^{2}}-\frac{\bar{\alpha}}{z-\bar{z}_{0}}- \\
& \frac{\bar{\alpha}\left(z_{0}-\bar{z}_{0}\right)\left(z+\bar{z}_{0}\right)}{\left(z-\bar{z}_{0}\right)^{3}}-\frac{\alpha \bar{z}_{0}}{\left(z-\bar{z}_{0}\right)^{2}}
\end{aligned}
$$

where $\alpha$ is the normalized Burger vector:

$$
\alpha=\frac{G\left(-i b_{x}+b_{y}\right)}{(\kappa+1) \pi}
$$

Note that at the interface only $b_{x}$, the $x$ component of the Burger's vector is nonzero while at the crack only $b_{y}$, the $y$-component of the Burger's vector is nonzero.

Let the stresses be written in the form $\sigma=\sigma_{p}+$ $\sigma_{h}+\sigma_{v}$ where $\sigma_{p}$ are the stresses due to the loading of perfectly bonded half-plane, $\sigma_{h}$ are the stresses duc to the dislocations distributed along the horizontal crack, and $\sigma_{v}$, are the stresses due to the dislocations distributed along the portions of the vertical interfaces, for $0<y<l,\left(l<h^{*}\right)$. This superposition can be written symbolically as:

$$
\sigma(z)=\sigma_{p}+\int_{0}^{l} \sigma_{v}\left(z, y_{0}\right) d y_{0}+\int_{0}^{d} \sigma_{h}\left(z, x_{0}\right) d x_{0}
$$

where $\sigma(z)$ is the total stress at some point $z$ in the domain. The interval 1 over which equation 7 is defined is an unknown

Imposing the traction-free condition over the opening zone and over the horizontal crack leads to a set of integral equations of the form:

$$
\sigma_{y p p}(x)+\frac{G}{\pi(\kappa+1)} \int_{0}^{1} b_{x}\left(y_{0}\right) Y_{v}\left(x, y_{0}\right) d y_{0}
$$

$$
\begin{gathered}
+\frac{G}{\pi(\kappa+1)} \int_{0}^{d} b_{r}\left(x_{0}\right) Y_{h}\left(x, x_{0}\right) d x_{0}=0 \\
\sigma_{x x p}(y)+\frac{G}{\pi(\kappa+1)} \int_{0}^{1} b_{x}\left(y_{0}\right) X_{v}\left(y, y_{0}\right) d y_{0} \\
+\frac{G}{\pi(\kappa+1)} \int_{0}^{d} b_{v}\left(x_{0}\right) X_{h}\left(y, x_{0}\right) d x_{0}=0
\end{gathered}
$$

The kernels for dislocation pairs are given by:

$$
\begin{aligned}
& X_{h}=+2 \operatorname{Re}\left(N_{1}-N_{2}\right)-\operatorname{Re}\left(P_{1}-P_{2}\right) \\
& X_{v}=-2 \operatorname{Im}\left(N_{1}+N_{2}\right)+\operatorname{Im}\left(P_{1}+P_{2}\right) \\
& Y_{h}=+2 \operatorname{Re}\left(N_{1}-N_{2}\right)+\operatorname{Re}\left(P_{1}-P_{2}\right) \\
& Y_{v}=-2 \operatorname{Im}\left(N_{1}+N_{2}\right)-\operatorname{Im}\left(P_{1}+P_{2}\right)
\end{aligned}
$$

where:

$$
\begin{aligned}
& N_{1} \pm N_{2}=\frac{1}{z-z_{0}} \pm \frac{1}{z-\bar{z}_{0}}+\frac{1}{\left(z+z_{0}\right)^{2}} \\
& {\left[\left(z_{0}-\bar{z}_{0}\right) \pm\left(z+z_{0}\right)\right] } \\
&-\frac{1}{\left(z-\bar{z}_{0}\right)^{2}}\left[\left(z-\bar{z}_{0}\right) \pm\left(\bar{z}_{0}-z_{0}\right)\right] \\
& P_{1} \pm P_{2}= \frac{1}{\left(z-z_{0}\right)^{2}}\left[\left(\bar{z}_{0}-\bar{z}\right) \mp\left(z-z_{0}\right)\right]+\frac{1}{\left(z+\bar{z}_{0}\right)^{2}} \\
& {\left[-\left(z+\bar{z}_{0}\right) \mp\left(z_{0}+\bar{z}_{0}\right)\right]+\frac{1}{\left(z+z_{0}\right)^{3}} } \\
& {\left[+\left(z+z_{0}\right)^{2}+\left(z-\bar{z}_{0}\right)\left(z-z_{0}-2 \bar{z}\right)\right] } \\
& \pm {\left[\left(z+z_{0}\right)\left(\bar{z}+z_{0}\right)\right]+\frac{1}{\left(z-\bar{z}_{0}\right)^{3}}\left[ \pm\left(z-\bar{z}_{0}\right)^{2}\right.} \\
&\left.+\left(z-\bar{z}_{0}\right)\left(z+\bar{z}_{0}-2 \bar{z}\right)\right]+\left[\left(z-\bar{z}_{0}\right)\left(\bar{z}-\bar{z}_{0}\right)\right]
\end{aligned}
$$

A standard collocation procedure introduced in [12] is used to solve the integral equations numerically. The unknown dislocation densities are interpolated in a continuous, piecewise linear fashion between discrete points. The values of the densities at the discrete points and the debond length I give the number of unknowns. The results to be shown are based on 25 unknowns along $x=a, 0<y<l$, and 25 unknowns along $0<x<d$, $y=h$.

From the results of the above analysis, the relationship between the applied load and the debond length for various geometrical parameters is established. Other quantities of interest are the relative 
displacement between the implant and the bone and the stresses at the bone-implant interface, both of which depend on the load in a nonlinear fashion.

\section{RESULTS AND DISCUSSION}

Some insight could be gained by analyzing the case of a perfectly bonded half-plane, i.e., a perfectly bonded implant. Because the normal stress $\sigma_{y y p}$, due to the applied traction $p$, decays with increasing depth, the longer the implant the smaller the normal stress experienced by the horizontal interface. Since we assume that the interfaces fail in tension, the horizontal interface will debond immediately, and the vertical interfaces would debond only after the normal stress $\sigma_{x x}$ at some point at the vertical interface reaches zero. Therefore, it would be helpful to analyze the case of a half-plane with an inevitably present horizontal subcrack under the traction $p$ (no vertical opening present, which is the scenario for relatively small ratios of $\left.p / \sigma_{\text {in }}\right)$.

Figure 3 demonstrates the effects of the presence of the horizontal crack on the normal stresses $\sigma_{\mathrm{xx}}$ at the vertical interfaces due to traction $p$ acting alone (with $\sigma_{\text {in }}=0$ ). Note that the dotted line gives the result for perfectly bonded half-plane (no horizontal crack present, or an infinitely long implant) and provides a basis for comparison.

For magnitudes of the dimensionless depth, $|y / a|$, less than about $h / 2$, the normal stresses for all combinations of horizontal crack depths and length are close to that for perfectly bonded case. As expected the results are closer to that for the perfectly bonded case for increasing $h$ and for decreasing $d$. With the

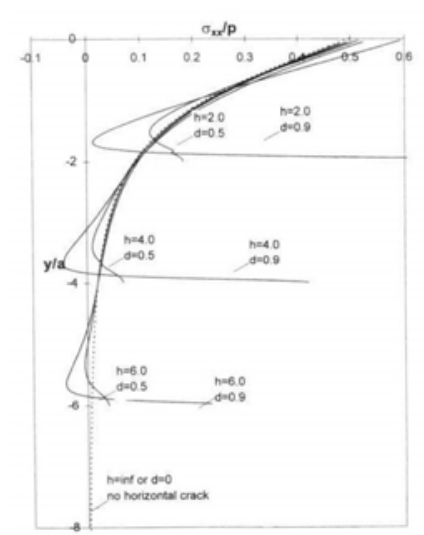

Fig. 3 Normal stress at the vertical interfaces, $\sigma_{x x}$, normalized by the applied traction $p$, in a presence of the horizontal crack of varying parameters $h$ and $d$. (no vertical opening and $\sigma_{\text {in }}=0$ ) dimensionless depth increasing, the changes in normal stress get larger. Specifically the stress decreases significantly (strengthening the interface), reaches the minimum, and than increases sharply close to the horizontal crack tip (weakening the interface). For example in the case of $h=6$ and $d=0.9$ the horizontal crack has in practice no effect on the normal stress for $|y / a|<4$; for $4<|y / a|<5.85$ it has the strengthening effect; and for $5.85<|y / a|<6$ the weakening effect is observed. The effects of the presence of the horizontal crack are increasing with $d$ for the same $h$. It also should be noted that the region of the weakening effect gets larger for smaller $d$.

Analyzing the results presented in Figure 3 leads to the conclusion that for large enough $p / \sigma_{\text {in }}$ at the bottom of the vertical interfaces (close to the horizontal crack tips) there will be regions where the total normal stress $\sigma_{x x}$ (due to traction $\mathrm{p}$ and compression $\sigma_{\text {in }}$ ) may be positive. However, when these regions coincide with the $\Delta h^{*}$ (see Figure 2), our assumption that the stress at the bone-implant interface, $0<|y / a|<h^{*}$, cannot be tensile is fulfilled. The presence of the horizontal crack could then be beneficial by increasing the compression at part of the interface.

It can also be concluded, that the vertical interfaces (failing in tension) will open at the top after $p / \sigma_{\text {in }}$ reaches a value of $1 / 0.5(=2)$ for infinitely long implant, and for $p / \sigma_{\text {in }}>1 / 0.6(=1.7)$ for very short implant, $h=2$. Again the opening from the bottom of the implant will not happen because of the tapered end with holes enabling for bone ingrowth (see Figure 1).

To further explain the issue of tensile stress developing in the vicinity of the horizontal crack tips, Figure 4 shows the normal stress, $\sigma_{x x}$, normalized by the applied traction $p$ at the vertical interfaces for

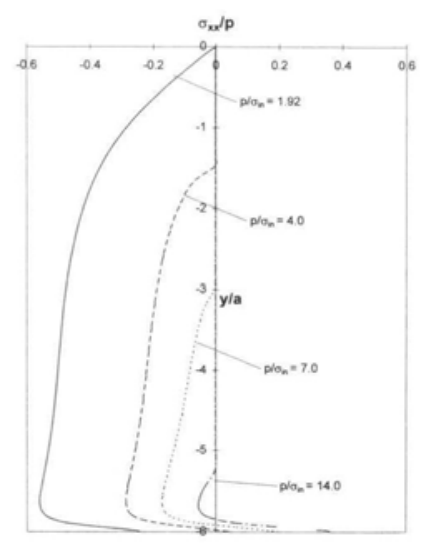

Fig. 4 Normal stress at the vertical interfaces, $\sigma_{x x}$, normalized by the applied traction $p$, for varying load $p / \sigma_{i n}$ (varying length of the vertical opening); normalized length of the implant $h=6$, normalized half-length of the crack $d=0.9$. 
various levels of normalized load $p / \sigma_{i n}$, in the case when they are large enough for the vertical openings to develop. The parameters $h=6$ and $d=0.9$ are typical of commercially available implants. Note that for $p / \sigma_{i n}=1.92(l=0)$ the $\sigma_{x x}$ is negative everywhere; for $p / \sigma_{i n}=4(l=1.53), \sigma_{x x}$ is tensile for $5.97<|y / a|<6$; for $p / \sigma_{\text {in }}=7(l=3.09)$, $\sigma_{x x}$ is tensile for $5.91<|y / a|<6$; and for $p / \sigma_{i n}=14(l=5.41), \sigma_{x x}$ is tensile for $5.83<$ $|y / a|<6$.

So, as discussed previously there are regions where the normal stress is tensile, but when those regions coincide with $\Delta h^{*}$, our assumption that the bone-implant interfaces do not experience tensile stress is fulfilled. It was found that taking $\Delta h^{*}=0.25$ for $d=$ 0.9 and $\Delta h^{*}=0.5$ for $\mathrm{d}=0.5$ satisfies the no tensile stress condition along the vertical interfaces for $0<$ $|y / a|<h^{*}$, and therefore in the results presented below this is assumed.

Figure 5 shows the shear stress, $\sigma_{x y}$, on the vertical interfaces normalized by the applied traction $p$. The thick solid line represents the case of perfectly bonded half-plane (no horizontal crack, no vertical openings), and provides a basis for comparison. The remaining four curves show the normalized shear stresses for varying levels of the applied load $p / \sigma_{i n}$ where the parameters are again $h=6$, and $d=0.9$.

Comparing the two solid lines (perfectly bonded case, horizontal crack present but no vertical opening) leads to the conclusion that the presence of the horizontal crack causes the shear stress to decrease except in the vicinity of the crack tips where the stress increases sharply. Comparing the remaining three curves (horizontal crack and vertical opening present) leads to the conclusion that the presence of the vertical openings alters the normalized shear stresses very

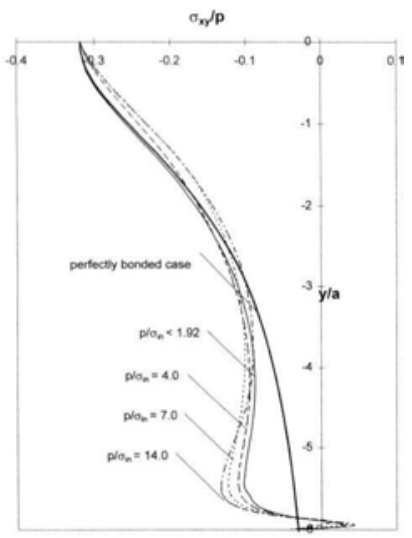

Fig. 5 Shear stress at the vertical interfaces, $\sigma_{x y}$, normalized by the applied traction $p$, for varying load $p / \sigma_{\text {in }}$ (varying length of the vertical opening); normalized length of the implant $h=6$, normalized half-length of the crack $d=0.9$. slightly. Thus, if the implant fails by shear, it will be at the surface where the shear stress is largest. The local increase in the shear stress at the distal end due to the presence of the horizontal crack does not reach the stress levels seen at the surface.

Figures 6-9 show the normalized length of the vertical opening as a function of the normalized load for various implant parameters. In Figure 6 the normalized length of the implant, $h$, is fixed at 4 , and in Figure 7 at 6 . The parameter $d$ takes values of 0.5 , $0.7,0.9$ and 1.0. The dotted lines represent the case when $d=0$ (no horizontal crack present). In Figure 8 the parameter $d$ is fixed at 0.9 and in Figure 9 at 0.5 . The implant length, $h$, takes values of 2,4 , and 6 . The dotted lines represent the case when $h$ is infinite (no horizontal crack present).

For relatively small loads, such that the $l$ s are small, the results for all the choices of the parameters are similar. This could be predicted by analyzing Figure 3: the normal stresses at the interfaces due to the applied traction $p$ alone, for all combinations of horizontal crack depth and length, being similar for $|\mathrm{y} / \mathrm{a}|<\boldsymbol{h}$. For middle range loads, the horizontal crack weakens the interface in the sense that less load is required for the same opening length; the effect increases with increasing $d$.

For large loads, such that $l \mathrm{~s}$ are close to $h^{*}$, the presence of the horizontal crack strengthens the interface, in the sense that more load is required to

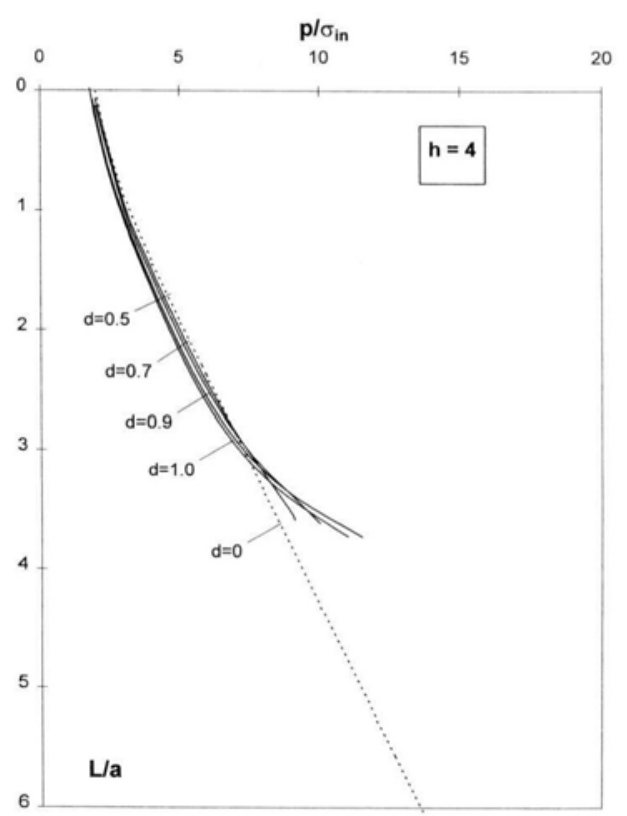

Fig. 6 Normalized length of the vertical opening $L / a$, as a function of the applied load $p / \sigma_{i n}$; normalized length of the implant, $h=4$. 


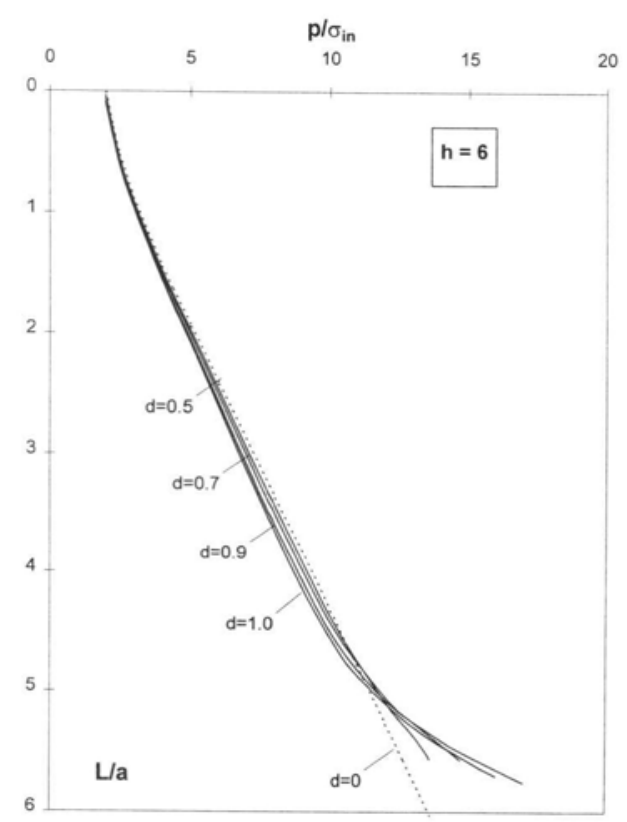

Fig. 7 Normalized length of the vertical opening $L / a$, as a function of the applied load $p / \sigma_{i n}$; normalized length of the implant, $h=6$.

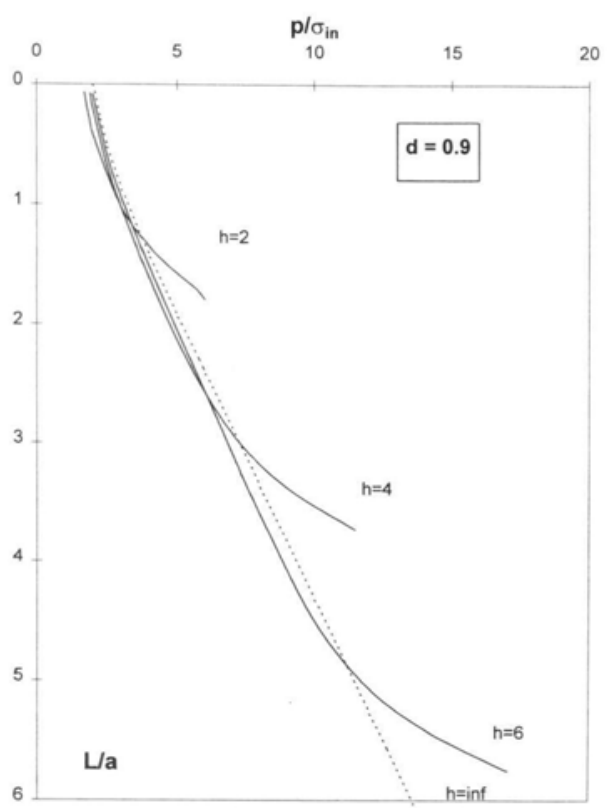

Fig. 8 Normalized length of the vertical opening $L / a$, as a function of the applied load $p / \sigma_{i n}$; normalized half-length of the crack, $d=0.9$. cause the same opening in the presence of the horizontal crack than without one (dotted lines). This effect increases with increasing $d$. Again this could be predicted by analyzing Figure 3-the normal stress due to traction $p$ alone, at part of the interface close to $h^{*}$ is smaller that one for the perfectly bonded case.

Figures 8 and 9 also provide some insight into the issue of maximum pull-out force dependence on implant diameter. The maximum pull-out force normalized by implant's length and remote stress is proportional to $\left(p / \sigma_{\text {in }}\right)_{\max } / h$. Our results show no variation of this normalized force with implant diameter for small d (Fig.9), and a $\sim 6 \%$ increase of force with a threefold increment of the diameter for large d (Fig.8).

In [13] the authors experimentally compared the immediate pull-out force of a large diameter implant $(4.5 \mathrm{~mm})$ with a standard size implant $(3.25 \mathrm{~mm})$. The maximum pull-out force was $16 \%$ greater for the larger implants, but was not statistically significant; similar to our results.

Figures 10-14 show the normalized relative displacement between the implant and the bone at the surface, as a function of the normalized load for various implant parameters. The normalized relative displacement is defined as follows:

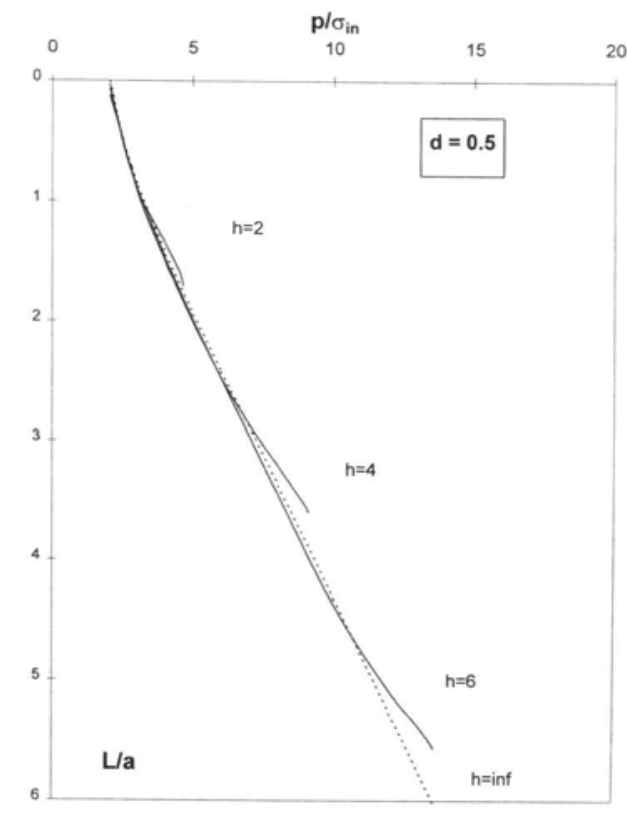

Fig. 9 Normalized length of the vertical opening $L / a$, as a function of the applied load $p / \sigma_{i n}$; normalized half-length of the crack, $d=0.5$. 


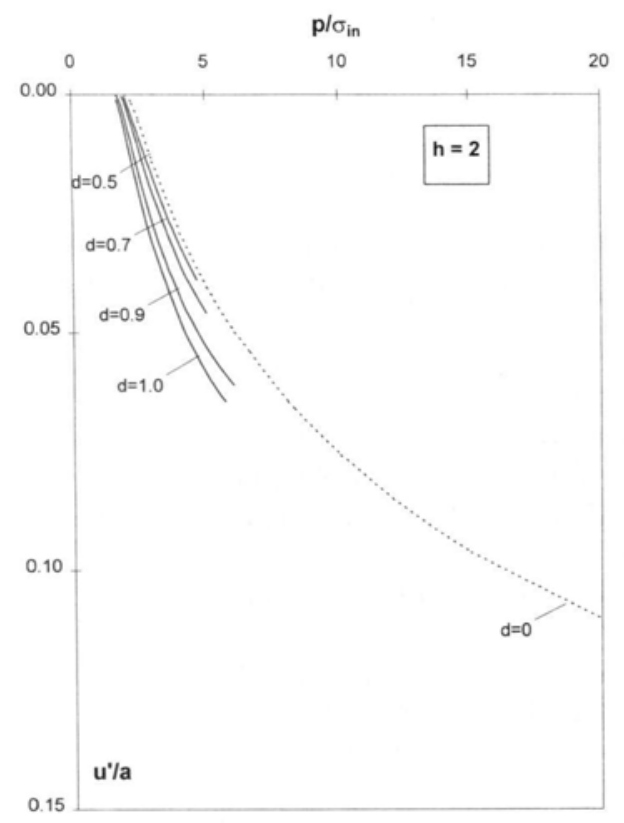

Fig. 10 Normalized surface gap between the implant and the bone, $u \% a$, as a function of the applied load $p / \sigma_{i n}$; normalized length of the implant, $h=2$.

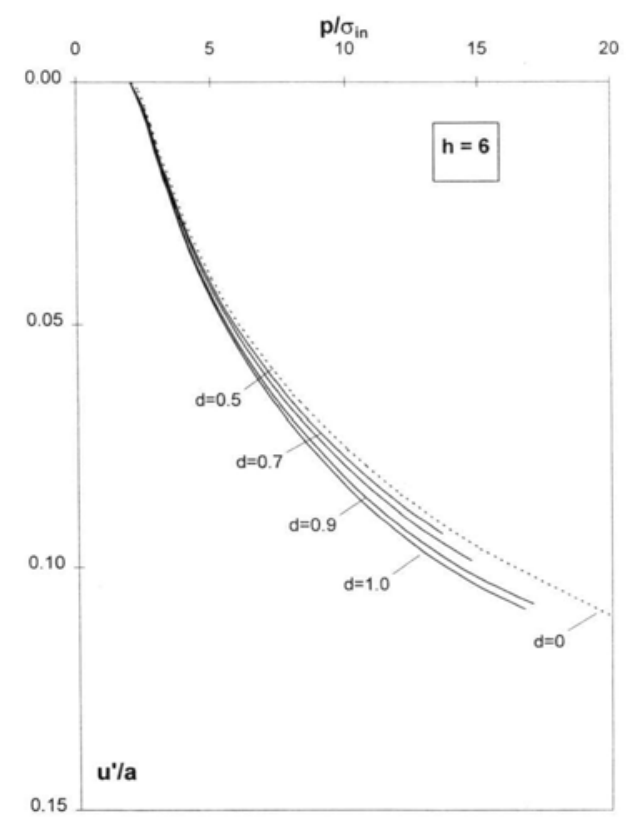

Fig. 11 Normalized surface gap between the implant and the bone, $u / a$, as a function of the applied load $p / \sigma_{\text {in }}$; normalized length of the implant, $h=4$.

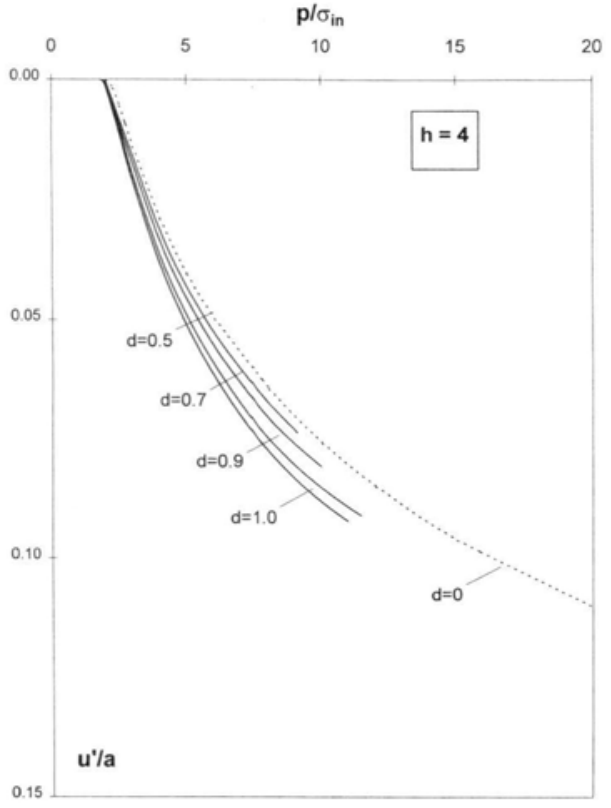

Fig. 12 Normalized surface gap between the implant and the bone, $u / a$, as a function of the applied load $p / \sigma_{i n}$; normalized length of the implant, $h=6$.

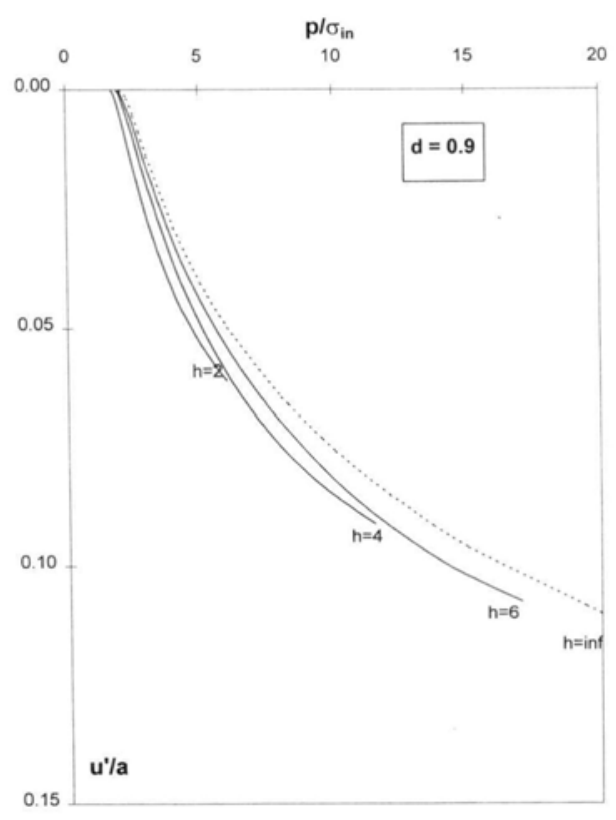

Fig. 13 Normalized surface gap between the implant and the bone, $u^{\prime} / a$, as a function of the applied load $p / \sigma_{i n}$; normalized half-length of the crack, $d=0.9$. 


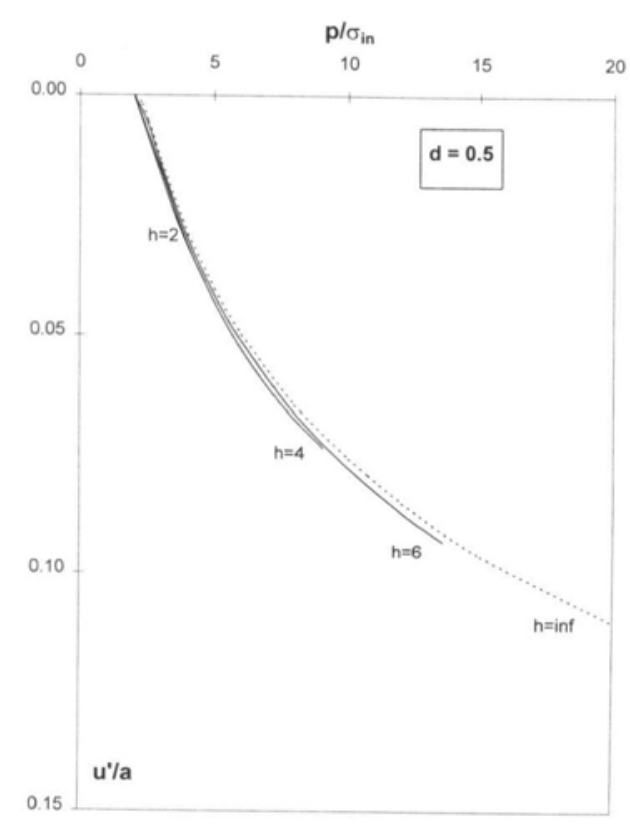

Fig. 14 Normalized surface gap between the implant and the bone, $u^{\prime} / a$, as a function of the applied load $p / \sigma_{i n}$; normalized half-length of the crack, $d=0.5$.

$$
\frac{u^{\prime}}{a}=\frac{u G}{\pi(\kappa+1) p a}
$$

In Figure 10 the length of the implant, $h$, is fixed at 2, in Figure 11 at 4 , and in Figure 12 at 6 . The parameter $d$ takes values of $0.5,0.7,0.9$ and 1.0 . The dotted lines represent the case when $d=0$ (no horizontal crack present). In Figure 13 the diameter $d$ is fixed at 0.9 , and in Figure 14 at 0.5 . The implant length, $\mathrm{h}$, takes values of 2,4 , and 6 . The dotted lines represent the case where $h$ is infinite (no horizontal crack present).

For a given implant length, $h$, and for a given normalized load, $p / \sigma_{\text {in }}$, the relative displacement at the surface, $\mathrm{u}^{\prime} / \mathfrak{a}$, increases with increasing diameter $d$. For a given diameter and for a given normalized load $\mathrm{u}^{\prime} / a$ decreases with increasing implant length $\mathrm{h}$.

\section{CONCLUSION}

For a given normalized implant length and normalized load, the surface gap increases with increasing normalized horizontal debond length. Also, for a given value of $d$ and $P$, the surface gap decreases with increasing $h$. Therefore, if the length of the debonded zone is crucial in the process of healing, the presence of horizontal debonding at the end of the implant could be in some instances beneficial. But, if the size of the gap at the surface is crucial in that process, the presence of the horizontal debonding worsens the situation.

Our analysis showed that the shear stress distribution along the vertical sides of the implant is not uniform. In fact, substantial local changes occur at the ends of the implant. Thus, in reducing experimental data, the common assumption of a uniform shear stress distribution would be inadequate and possibly lead to erroneous conclusions. This point is reinforced in [14].

Theoretical models like the one presented here can provide insight into the possible mechanisms of failure of dental implants. The applicability of this model goes beyond the area of dental implants. For example, this model could provide a basis for discussion of the pullout failure of bone screws. The no-slip boundary conditions provide a rough approximation to the conditions present between the screw threads and the surrounding bone.

\section{REFERENCES}

1. Gottlander, M., Albrektsson, T., and Carlsson, L.V., "A Histomorphometric Study of Unthreaded Hydroxyapatite-Coated and Titanium-Coated Implants in Rabbit Bone," The International Journal of Oral \& Maxillofacial Implants, Vol. 7, No. 4, pp. 485-490, 1992.

2. Albrektsson, T., "Direct Bone Anchorage of Dental Implants," The Journal of Prosthetic Dentistry, Vol. 50, No. 2, pp. 255-261, 1983.

3. Kay, J.F., "'Material' Changes in Engineering Dental Implants," SOMA, pp. 36-41, January, 1988.

4. Kraut, R.A., Dootson, J., and McCullen, A., "Biomechanical Analysis of Osseointegration of IMZ Implants in Goat Mandibles and Maxillae," The International Journal of Oral \& Maxillofacial Implants, Vol. 6, No. 2, pp. 187-194, 1991.

5. Mihalko, W.M., May, T.C., Kay, J.F., and Krause, W.R., "Finite Element Analysis of Interface Geometry Effects on the Crestal Bone Surrounding a Dental Implant," Implant Dentistry, Vol. 1, No. 3, pp. 212-217, 1992.

6. Dollar, A., and Steif, P.S., "Load Transfer in Composites with a Coulomb Friction Interface," Int. J. Solids Struct., Vol. 24, 1988, pp. 789-803.

7. Clift, S.E., Fisher, J, and Watson, C.J., "Finite Element Stress and Strain Analysis of the Bone Surrounding a Dental Implant: Effect of Variations in Bone Modulus," Journal of Engineering in Medicine, Vol. 206, No. 4, pp. 233-241, 1992. 
8. Mak, A.F., Keer, L.M., Chen, S.H., and Lewis, J.L., "A No-Slip Interface Crack," ASME Journal of Applied Mechanics, Vol. 102, 1980, pp. 347-350.

9. Keer, L.M., and Meade, K.P., "A Note on a No-Slip Interface Crack," ASME Journal of Applied Mechanics, Vol. 49, 1982, pp. 454-455.

10. Clech, J.P., Keer, L.M., and Lewis, J.L., "An Array of No-Slip Tensile Cracks at a Bimaterial Interface," Engineering Fracture Mechanics, Vol. 23, 1986, 899-911.

11. Muskhelishvili, N.I., Some Basic Problems of the Mathematical Theory of Elasticity, 1963, Noordhoff, The Netherlands.
12. Erdogan, F., Gupta, G.D., and Cook, T.S., "Numerical Solution of Singular Integral Equations," in Mechanics of Fracture I. Methods of Analysis and Solutions of Crack Problems, Sih, G. C., ed., 1973, Noordhoff, The Netherlands.

13. Kido, H., Kumar, A, Schulz, E.E., Lozada, J. and Saha, S., "Biomechanical Evaluation of the Stability of Small and Large diameter Implants: Preliminary Study," IEEE Proceedings of the 15th Southern Biomedical Engineering Conference, March 29-31, 1996.

14. Berzins, A., Shah, B. Weinans, H., and Sumner, D.R., "Nondestructive Measurements of ImplantBone Interface Shear Modulus and Effects of Implant Geometry in Pull-Out Tests," J. Biomedical Materials Research, in press, 1997. 